FFIA-UV/03-01

\title{
NUCLEATION OF ${ }^{(4)} R$ BRANE UNIVERSES
}

\author{
Rubén Cordero $^{a *}$ and Efraín $\operatorname{Rojas}^{b \dagger}$ \\ a Departamento de Física, Escuela Superior de Física y Matemáticas del I.P.N. \\ Unidad Adolfo López Mateos, Edificio 9, 07738 México, D.F., MEXICO \\ ${ }^{b}$ Facultad de Física e Inteligencia Artificial, Universidad Veracruzana \\ Sebastián Camacho 5; Xalapa, Veracruz; 91000, MEXICO
}

December 25, 2018

\begin{abstract}
The creation of brane universes induced by a totally antisymmetric tensor living in a fixed background spacetime is presented, where a term involving the intrinsic curvature of the brane is considered. A canonical quantum mechanical approach employing Wheeler-DeWitt equation is done. The probability nucleation for the brane is calculated taking into account both an instanton method and a WKB approximation. Some cosmological implications arose from the model are presented.
\end{abstract}

\footnotetext{
*Email: cordero@fis.cinvestav.mx

†Email: efrojas@uv.mx
} 


\section{Introduction}

Nowadays, with the standard cosmology the famous fundamental question, "where did it all come from?" still it does not have a convincing answer, reason why a new description is necessary. Cosmologists during long time have believed that quantum cosmology can shed light on this question [1, 2, 3, 4, but some issues are in controversy, e.g. the lack of an intrinsic time variable in the theory [5], the validity of the minisuperspace approximation, the problem of cosmological boundary conditions [6], to mention something. Among the proposals trying to outline a possible answer to the fundamental question, the so-called Brane World Scenaries (BWS) [7, 8] became a promising way to understand the birth and then the evolution of our Universe. Grounded on the proposal that our universe can be thought as a 4-dimensional spacetime object embedded in an $\mathrm{N}$-dimensional spacetime, the main physical idea behind of BWS is that the matter fields are confined to a 3-dimensional space (brane) while the gravitational fields can extend into a higher-dimensional space (bulk), where the graviton can travel into the extra dimensions. Originally proposed to resolve the hierarchy problem, BWS has been applied to a great diversity of situations such as dark matter/energy, quientessence, cosmology, inflation and particle physics. On other hand, at the formal mathematical level, related applications of embedding theory such as generation of internal symmetries, quantum gravity and alternative Kaluza-Klein theories have been exploited [9, 10, 11, 12, 13. In the cosmology context there are predictions of these ideas, that could be tested by astronomical observations what constitutes one of the several reasons for which it is so attractive, so that it has predictive power [14].

In these brane world programs, gravity on the brane can be recovered by compactifying the extra dimensions [7] or by introducing an AdS background spacetime [8]. However, Dvali, Gabadadze and Porrati [15] (DGP) showed that, even in an asymptotically Minkowski bulk, 4-dimensional gravity can be recovered if one includes a brane curvature term in the action. Furthermore, DGP considered the $Z_{2}$ reflection symmetry with respect to the brane getting that gravity, is 4-dimensional on smaller scales than a certain scale, or it is 5-dimensional on larger distances [16, 17]. It is noteworthy that reflection symmetry is not the only possibility in these models. With regard to the last, several works have been devoted to antisymmetric cases [18, 19, 20, 21, 22, 23, 24, 25, for instance, when the brane is coupled to a 4 -form field [23]. In a pionner work, Brown and Teitelboim worked out the process of membrane creation by an antisymmetric field motivated by Schwinger process of pair creation induced for the presence of a electric field [26]. Garriga [27] has also studied the creation of membranes for this field in a dS background. Others authors have been interested in brane world creation in AdS spacetime or in other particular situations [28, 29, 30, 31] but, upon our knowledge, 
nobody has been devoted to the nucleation of Brane World Universes (BWU) induced by a 4 -form field besides a brane curvature term included in the action. Generally, BWS are studied mostly for AdS/dS as well as empty (Minkowski) backgrounds.

In this paper we are going to discuss the nucleation of BWU with a curvature term induced by a 4 -form field in a dS background spacetime. We get the Friedman like equation when 5-dimensional gravity is fixed and perform geometric Hamiltonian analysis in order to obtain, by means of canonical quantization, the corresponding Wheeler-DeWitt equation. The setup for the induced brane production is as follows. There is an external homogeneous field that produces a brane; then, the natural question there, is: what is the probability of such process? In the present paper we calculate the creation probability for a brane universe embedded in a de Sitter space, produced by a 4-form potential gauge field in the same way that the standard electromagnetic potential bears to a charged particle. In its quantum analisys we shall use a WKB approximation attaining the same results by an instanton method. We could try to answer the question of which one of the universes arose is the more probable universe produced in this model and if our Universe is one of them, or could be a very special universe. Parameters of this model must be constrained by cosmological requirements like nucleosynthesis [23].

The paper is organized as follows. In Sec. II we present the equations of motion of a brane with matter and curvature term that lives in a AdS/dS or Minkowski bulk when there is no $Z_{2}$ symmetry and, by means of a limit equivalent to the presence of a 4 form field in a fixed background the corresponding equations. A geometric Hamiltonian approach is done in Sec. III, where the fundamental canonical structure is obtained and the canonical constraints are listed. The next step is specialize the general canonical analysis to the case of a spherical 3-brane floating in an $\mathrm{dS}_{5}$ background spacetime which is the issue of Sec. IV. The last provides the preamble to obtain the WdW equation in the canonical quantization context, which is done in Sec. V. The creation probability is calculated in Sec. VI by two methods, the first is an instanton approach and the other one by means of a WKB approach for barrier tunneling of the WdW equation. Finally in Sec. VII, we present our conclusions as well as some perspectives of our work.

\section{The model}

The effective action that we are interested in the brane world model corresponds to a 3-brane with a intrinsic curvature term considered from its worldsheet and no $Z_{2}$

symmetry in the presence of a fixed background spacetime. We consider the following 
action

$$
S=\int d^{5} y \sqrt{-g}\left(\frac{1}{2 k}{ }^{(5)} \mathcal{R}+\mathcal{L}_{m}\right)+\int d^{4} x \sqrt{-\gamma}\left(\frac{1}{2 k^{\prime}} \mathcal{R}-L_{m}\right)
$$

where $\mathcal{L}_{m}$ and $L_{m}=\rho_{v}$ stand for matter Lagrangians for the bulk and the brane, respectively. In our case, we will consider those as cosmological constants. The constants $k=M_{(N)}^{2-N}$ and $k^{\prime}=M_{(4)}^{-2}$, where $M_{(4)}$ and $M_{(N)}$ are the brane Plank and bulk masses. $N$ denotes the dimension of the bulk. The respective equations of motion for the brane are [19],

$$
\begin{aligned}
{[K] \gamma_{a b}-\left[K_{a b}\right] } & =k \mathcal{T}_{a b} \\
\widetilde{T}^{a b}<K_{a b}> & =\left[\mathcal{T}_{n n}\right] \\
\nabla_{a}\left(T^{a}{ }_{b}\right) & =-\left[\widetilde{T}_{b n}\right] .
\end{aligned}
$$

where $K_{a b}$ is the extrinsic curvature of the brane, $\gamma_{a b}$ denotes the worldsheet metric. $\mathcal{T}_{\text {ab }}=\left(\mathcal{T}_{\text {bulk }}\right)_{\mu \nu} e^{\mu}{ }_{a} e^{\nu}{ }_{b}, \mathcal{T}_{\text {an }}=\left(\mathcal{T}_{\text {bulk }}\right)_{\mu \nu} e^{\mu}{ }_{a} n^{\nu}$ and $\mathcal{T}_{n n}=\left(\mathcal{T}_{\text {bulk }}\right)_{\mu \nu} n^{\mu} n^{\nu}$ are the projections onto the worldsheet of the bulk energy-momentum tensor. The square and angular brackets represent the difference and the average of the corresponding embraced quantity, on the two sides of the brane, respectively, i.e., $\left[K_{a b}\right]=K_{a b}^{+}-K_{a b}^{-}$and $\left\langle K_{a b}\right\rangle=$ $\frac{1}{2}\left(K_{a b}^{+}+K_{a b}^{-}\right)$, where ' + ' and '-' denote the exterior and interior of the brane.

Taking into account that the bulk energy momentum tensor has the form

$$
\mathcal{T}_{\mu \nu}^{ \pm}=-k^{-1} \Lambda^{ \pm} g_{\mu \nu}
$$

and by means of the generalized Birkhof theorem, the 5-dimensional FRW metric can be written as

$$
d S_{5}^{2}=-A_{ \pm} d \tau^{2}+A_{ \pm}^{-1} d a^{2}+a^{2} d \Omega_{3}^{2}
$$

where

$$
A_{ \pm}=\kappa-\frac{\Lambda^{ \pm}}{6} a^{2}-\frac{2 \mathcal{M}^{ \pm}}{M_{(5)}^{3} a^{2}},
$$

and $d \Omega_{3}^{2}$ denotes the metric of a 3 -sphere, $a$ is the cosmic scale factor and $\mathcal{M}^{ \pm}$is the mass. Furthermore, in the cosmic time gauge the 4-dimensional metric on the brane reduces to

$$
d S_{4}^{2}=-d t^{2}+a^{2} d \Omega_{3}^{2}
$$

Using the junction conditions, and due to we have isotropy and homogeneity in (2.6), matter can be parametrized completely via a perfect fluid brane energy-momentum tensor

$$
T_{b}^{a}=\operatorname{diag}(-\rho, P, P, P),
$$


so the relevant equations of motion for the model are the following

$$
\begin{aligned}
\left(\dot{a}^{2}+A_{-}\right)^{1 / 2}-\left(\dot{a}^{2}+A_{+}\right)^{1 / 2} & =\frac{k a}{3}\left(\rho-\frac{3\left(\dot{a}^{2}+1\right)}{k^{\prime} a^{2}}\right), \\
\dot{\rho}+3 \frac{\dot{a}}{a}(\rho+P) & =0 .
\end{aligned}
$$

Last equation represents the energy-momentum conservation on the brane. The former system was discussed in 32 where several interesting cases were treated. Suppose now $\mathcal{M}^{-}=0, \rho=$ const, and consider at the same time, the limits of fixed bulk gravity, $M_{(5)} \rightarrow \infty$ and, $\Lambda^{+} \rightarrow \Lambda^{-}$but satisfying the following relation

$$
\operatorname{Lim}_{\left(M_{(5)}, \Lambda^{+}\right) \rightarrow\left(\infty, \Lambda^{-}\right)}\left(\Lambda^{+}-\Lambda^{-}\right) M_{(5)}^{3}=\alpha,
$$

so, expanding the second term of the LHS of Eq. (2.11), this equation transform to

$$
\left(\frac{\rho}{3}-M_{(4)}^{2} \frac{\dot{a}+1}{a^{2}}\right)\left(\frac{\dot{a}+1}{a^{2}}-\frac{\Lambda}{6}\right)^{1 / 2}=\frac{\alpha}{12}+\frac{\mathcal{M}}{a^{4}} .
$$

In order to get the Friedman like equation we define a $\Upsilon$ quantity through its definition

$$
\frac{\dot{a}+1}{a^{2}} \equiv \frac{\rho}{3 M_{(4)}^{2}} \Upsilon \equiv H^{2} \Upsilon .
$$

Note that $\Upsilon$ is only a function of $a$ and it is a solution of the following relation

$$
M_{(4)}^{4}(1-\Upsilon)^{2}\left(\Upsilon-\frac{\Lambda}{6 H^{2}}\right)=H^{-6}\left(\frac{\alpha}{12}+\frac{\mathcal{M}}{a^{4}}\right)^{2}
$$

As we will see below, this approach is equivalent to a brane interacting with a 4-form field and propagating in a fixed background spacetime.

\section{Hamiltonian Approach}

The Hamiltonian framework has been a fundamental prop in the study of the dynamics of field theories besides of appoint oneself a preliminary step towards canonical quantization in physical theories. Knowingly of previous fact, canonical quantization is the oldest and most conservative approach to quantization which we would like to develop in order to attain the quantum cosmology emerged from our BWU model. To carry out the previous thing, we must begin by casting the theory in a canonical fashion, then we shall proceed to its quantization.

To begin with, we are going to mimic the well known ADM procedure for canonical gravity to get a hamiltonian description of the brane. We shall assume that the worldsheet $m$ admits a foliation, i.e., we will begin with a time like 4-manifold $m$ topologically 
$\Sigma \times R$, equipped with a metric $\gamma_{a b}$, such that $m$ is an outcome of the evolution of a space like 3-manifold $\Sigma_{t}$, representing "instants of time", each of which is diffeomorphic to $\Sigma$. Then we shall proced to identify the several geometric quantities inherent to the hypersurface $\Sigma_{t}$. The ADM decomposition of the action, computation of the momenta as well as the recognition of the constraints are the succesive stages.

\subsection{Model ADM decomposed}

Leaning in results achieved in 34, 35, 36, we are going to display the standard procedure. We start considering the action

$$
S=\frac{k_{1}}{2} \int_{m} \sqrt{-\gamma}\left(\mathcal{R}+\Lambda_{b}\right)+\frac{k_{2}}{4 !} \int_{m} \sqrt{-\gamma} A_{\mu \nu \rho \sigma} \epsilon^{\mu \nu \rho \sigma},
$$

where $\mathcal{R}$ is the Ricci scalar curvature of the worldsheet $m, k_{1}=M_{(4)}^{2}$ and $\Lambda_{b}=-2 \rho_{v} / M_{(4)}^{2}$ being the cosmological constant on the brane. $A_{\mu \nu \rho \sigma}$ is a gauge 4-form Ramond-Ramond field onto the bulk, $\mu, \nu=0,1, \ldots, N-1 . \epsilon^{\mu \nu \rho \sigma}$ is an antisymmetric bulk tensor which can be expressed in terms of the worldsheet Levi-Civita tensor as $\epsilon^{\mu \nu \rho \sigma}=\epsilon^{a b c d} e^{\mu}{ }_{a} e^{\nu}{ }_{b} e^{\rho}{ }_{c} e^{\sigma}{ }_{d}$, where $e^{\mu}{ }_{a}$ denotes the tangent vectors to the worldsheet, $a, b=0,1,2,3 . \quad k_{2}$ is the coupling constant between the brane and the antisymmetric tensor.

Before going on, we would like to glimpse onto the ADM decomposition of some important geometric quantities defined onto the branes in our geometrical approach. In the Appendix we have included notation and some important facts for embedding theories to have reference of the material useful through the paper.

Taking into account the Gauss-Codazzi relations for the embedding of $\Sigma_{t}$ in $m$, Eqs. (A.4) and (A.5), up to a divergence term we have an equation involving the curvatures either extrinsic and intrinsic

$$
\mathcal{R}=R+\left(k_{A B} k^{A B}-k^{2}\right),
$$

where $R$ denotes the intrinsic curvature* of $\Sigma_{t}$ which does not have any dependence of the velocity and $k_{A B}$ its extrinsic curvature associated with the unit timelike normal $\eta^{\mu}$, given by

$$
\begin{aligned}
k_{A B} & =-g_{\mu \nu} \eta^{\mu}\left(\mathcal{D}_{A} \epsilon_{B}^{\nu}+\Gamma_{\alpha \beta}^{\mu} \epsilon_{A}^{\alpha} \epsilon_{B}^{\beta}\right) \\
& :=-g_{\mu \nu} \eta^{\mu} \tilde{\mathcal{D}}_{A} \epsilon_{B}^{\nu} .
\end{aligned}
$$

*We will adhere to Wald's convention concerning the definitions of Riemannian curvature, namely, $2 \nabla_{[a} \nabla_{b]} t^{c}=-\mathcal{R}_{a b d} t^{d}[37$ 
Besides of (3.3), in $\Sigma_{t}$ we have another curvature tensor associated with the $i$ th unit normal $n^{\mu i}$

$$
K_{A B}^{i}=-g_{\mu \nu} n^{\mu i} \tilde{\mathcal{D}}_{A} \epsilon^{\nu},
$$

where $g_{\mu \nu}$ denotes the background spacetime metric and $i=1,2, \ldots, N-d ; A, B=$ $1,2,3$. Note that the configuration space consists of the embedding functions $X^{\mu}$ for the brane, instead of 3-metrics as is customary in the ADM approach for general relativity.

In order to simplify the computations below, the next relations will be more useful since the velocities appear explicitly

$$
\begin{aligned}
\kappa_{A B} & =N k_{A B} \\
& =-g_{\mu \nu} \dot{X}^{\mu} \tilde{\mathcal{D}}_{A} \epsilon_{B}^{\nu} .
\end{aligned}
$$

For canonical purposes will be useful the next time derivative

$$
\frac{\partial N}{\partial \dot{X}^{\mu}}=-\eta_{\mu}=-g_{\mu \nu} \eta^{\nu}
$$

As before, we will need the derivatives of the extrinsic curvature

$$
\begin{aligned}
\frac{\partial \kappa_{A B}}{\partial \dot{X}^{\mu}} & =-g_{\mu \nu} \tilde{\mathcal{D}}_{A} \epsilon_{B}^{\nu} \\
& =-k_{A B} \eta_{\mu}+K_{A B}^{i} n_{\mu i}
\end{aligned}
$$

where in the second line on the RHS we have used the Gauss-Weingarten equations (A.1).

The ADM decomposed action (3.1) now looks like

$$
S=\int_{\Sigma_{t}} \int_{R} \frac{k_{1}}{2} N \sqrt{h}\left[\bar{R}+k_{A B} k^{A B}-k^{2}\right]+\int_{\Sigma_{t}} \int_{R} \frac{k_{2}}{3 !} A_{\mu \nu \rho \sigma} \dot{X}^{\mu} \epsilon^{\nu}{ }_{A} \epsilon_{B}^{\rho} \epsilon_{C}^{\sigma} \varepsilon^{A B C}
$$

where we have defined $\bar{R}:=R+\Lambda_{b}$ and $h$ is the determinant of the hypersurface metric $h_{A B}$ and $\varepsilon^{A B C}$ is the $\Sigma_{t}$ Levi-Civita antisymmetric symbol.

\subsection{Primordial tensor}

We define for convenience the following symmetric tensor which is independent of the velocities

$$
\begin{aligned}
\Theta^{\mu}{ }_{\nu} & :=\left(h^{A B} h^{C D}-h^{A C} h^{B D}\right) \tilde{\mathcal{D}}_{A} \epsilon^{\mu}{ }_{B} \tilde{\mathcal{D}}_{C} \epsilon_{\nu D} \\
& =\left(k^{2}-k_{A B} k^{A B}\right) \eta^{\mu} \eta_{\nu}-\left(k L^{i}-K_{A B}^{i} k^{A B}\right) n^{\mu}{ }_{i} \eta_{\nu} \\
& -\left(k L^{i}-K_{A B}^{i} k^{A B}\right) \eta^{\mu} n_{\nu i}+\left(L^{i} L^{j}-K_{A B}^{i} K^{A B j}\right) n^{\mu}{ }_{i} n_{\nu j},
\end{aligned}
$$


where $L^{i}$ denotes the trace of the curvature $K_{A B}^{i}$, i.e., $L^{i}=h^{A B} K_{A B}^{i}$. This tensor will keep track of the dynamics of the theory as we will below. The tensor (3.9) was previously defined in 33] where a Hamiltonian analysis for geodetic brane gravity was performed. We will have in mind some ideas of the classical approach developed there.

Some of the important properties we are interested from the tensor (3.9) are the following

$$
\begin{aligned}
\Theta^{\mu}{ }_{\alpha} \epsilon_{A}^{\alpha} & =0 \\
\Theta^{\mu}{ }_{\alpha} \dot{X}^{\alpha} & =-N\left(k^{2}-k_{A B} k^{A B}\right) \eta^{\mu}+N\left(k L^{i}-K_{A B}^{i} k^{A B}\right) n^{\mu}{ }_{i}, \\
g_{\mu \nu} \dot{X}^{\mu} \Theta^{\nu}{ }_{\alpha} \dot{X}^{\alpha} & =N^{2}\left(k^{2}-k_{A B} k^{A B}\right) .
\end{aligned}
$$

We shall adopt the notation $\dot{X} \cdot \Theta \cdot \dot{X}:=g_{\mu \nu} \dot{X}^{\mu} \Theta^{\nu}{ }_{\alpha} \dot{X}^{\alpha}$ throughout the paper. Taking advantage of the previous results we are able to rewrite the Lagrangian density as follows

$$
\mathcal{L}=\frac{k_{1}}{2} N \sqrt{h}\left[\bar{R}-\frac{1}{N^{2}} \dot{X} \cdot \Theta \cdot \dot{X}\right]+\frac{k_{2}}{3 !} A_{\mu \nu \rho \sigma} \dot{X}^{\mu} \epsilon^{\nu}{ }_{A} \epsilon^{\rho}{ }_{B} \epsilon_{C}^{\sigma} \varepsilon^{A B C} .
$$

Using the tensor (3.9), the momenta associated to the embedding functions are the following

$$
\begin{aligned}
P_{\mu} & =\frac{\partial \mathcal{L}}{\partial \dot{X}^{\mu}} \\
& =-\frac{k_{1}}{2} \sqrt{h}\left\{\left[\bar{R}+\frac{1}{N^{2}} \dot{X} \cdot \Theta \cdot \dot{X}\right] \eta_{\mu}+\frac{2}{N} \Theta_{\mu \nu} \dot{X}^{\nu}\right\}+\frac{k_{2}}{3 !} A_{\mu \alpha \beta \gamma} \bar{\varepsilon}^{\alpha \beta \gamma},
\end{aligned}
$$

where we have defined the $\Sigma_{t}$-antisymmetric tangent tensor $\bar{\varepsilon}^{\mu \nu \rho}=\varepsilon^{A B C} \epsilon^{\mu}{ }_{A} \epsilon^{\nu}{ }_{B} \epsilon_{C}^{\rho}$ with normalization $\bar{\varepsilon}^{\mu \nu \rho} \bar{\varepsilon}_{\mu \nu \rho}=3$ ! .

\subsection{Canonical Constraints}

Due to we have in hands an invariant reparametrization theory, a natural question to ask is what its inherited primary constraints are. This is part of the chore for constrained field theories. According to the standard Dirac-Bergmann algorithm, we will get the constraints from the momenta (3.11). It is convenient for the computation, define the matrix $\Psi^{\mu}{ }_{\nu}:=\Theta^{\mu}{ }_{\nu}-\lambda g^{\mu}{ }_{\nu}$ where $\lambda(x)$ is a not dynamical field which is gauge dependent 33, to be found. If we assume that the form of momenta have the following pattern,

$$
P_{\mu}=-\sqrt{h} k_{1}(\Theta-\lambda g)_{\mu \nu} \eta^{\nu}+\frac{k_{2}}{3 !} A_{\mu \alpha \beta \gamma} \bar{\varepsilon}^{\alpha \beta \gamma},
$$

we are free to compare both expressions (3.11) and (3.12) to get a condition to be satisfied

$$
\bar{R}+\eta \cdot \Theta \cdot \eta+2 \lambda=0
$$


This expression will metamorphose in a primary constraint after we express it in terms of phase space variables.

Profitable is the introduction of the field $\lambda(x)$ since we can solve Eq.(3.12) for the timelike unit normal vector

$$
\eta^{\mu}=\frac{-1}{\sqrt{h} k_{1}}\left(\Psi^{-1}\right)^{\mu}{ }_{\alpha}^{\alpha \beta} \mathcal{P}_{\beta}
$$

where we have defined $\mathcal{P}_{\mu}=P_{\mu}-\frac{k_{2}}{3 !} A_{\mu \alpha \beta \gamma} \bar{\varepsilon}^{\alpha \beta \gamma}$, but we have to pay a price which is enlarge the number of constraints as we will see below. Inserting this form of the unit time-like vector in the relation (3.13), we get the main scalar primary constraint. In a similar way, inserting $\eta^{\mu}$ in its square relation, $g(\eta, \eta)=-1$, we have another scalar constraint.

The complete set of primary constraints we have in hand are the following

$$
\begin{aligned}
C_{0} & =\mathcal{P} \cdot\left(\Psi^{-1}\right) \cdot \mathcal{P}+h \lambda_{0} k_{1}^{2}=0, \\
\mathcal{C}_{0} & =\mathcal{P} \cdot\left(\Psi^{-2}\right) \cdot \mathcal{P}+h k_{1}^{2}=0, \\
C_{A} & =\mathcal{P}_{\mu} \epsilon^{\mu}{ }_{A}=0, \\
\mathcal{C}_{\lambda} & =P_{\lambda}=0,
\end{aligned}
$$

where we have defined $\lambda_{0}=\lambda+\bar{R}$. The third constraint is the always inherited constraint to the parametrized theories while the last one came from the fact that $\lambda$ is not a dynamical field, i.e., its time derivative does not appear in the Lagrangian. It is worthy mention that the constraint $\mathcal{C}_{0}$ is a byproduct of $C_{0}$ taking advantage of the identity $\partial\left(\Psi^{-1}\right)^{\mu}{ }_{\nu} / \partial \lambda=\left(\Psi^{-2}\right)^{\mu}{ }_{\nu}$.

\section{Brane Universe Floating in a de Sitter Space}

The main idea in this section is adapt the previous dynamical description to the case of a spherical brane immersed in a specific background spacetime in order to apply the quantum approach to our BWS model.

Consider a 3-dimensional spherical brane evolving in a de Sitter 5-dimensional background spacetime, $d S_{5}^{2}=-A_{ \pm} d \tau^{2}+A_{ \pm}^{-1} d a^{2}+a^{2} d \Omega_{3}^{2}$, where $A_{ \pm}$is given by (2.7). The worldsheet generated by the motion of the brane can be described by the following 
embedding

$$
x^{\mu}=X^{\mu}(\tau, \chi, \theta, \phi)=\left(\begin{array}{c}
t(\tau) \\
a(\tau) \\
\chi \\
\theta \\
\phi
\end{array}\right) .
$$

The line element induced on the worldsheet is given by

$$
d s^{2}=\left(-A_{ \pm} \dot{t}^{2}+A_{ \pm}^{-1} \dot{a^{2}}\right) d \tau^{2}+a^{2} d \chi^{2}+a^{2} \sin ^{2} \chi d \theta^{2}+a^{2} \sin ^{2} \chi \sin ^{2} \theta d \phi^{2}
$$

where the dot stands for derivative with respect to cosmic time $\tau$. For convenience in notation we define $\Delta=-A_{ \pm} \dot{t}^{2}+A_{ \pm}^{-1} \dot{a}^{2}$. The frecuently appealed cosmic gauge will be set up by $\Delta=-1$.

In order to evaluate the extrinsic curvature tensors involved in our approach, (3.3) and (3.4), we need the orthonormal $\Sigma_{t}$ basis

$$
\eta^{\mu}=\frac{1}{\sqrt{-\Delta}}(\dot{t}, \dot{a}, 0,0,0), \quad n^{\mu}=\frac{1}{\sqrt{-\Delta}}\left(A_{ \pm}^{-1} \dot{a}, A_{ \pm} \dot{t}, 0,0,0\right)
$$

The only nonvanishing components for the extrinsic curvatures are

$$
\begin{array}{rlrl}
k_{\chi \chi} & =\frac{a \dot{a}}{(-\Delta)^{1 / 2}} & K_{\chi \chi} & =\frac{a \dot{t}}{(-\Delta)^{1 / 2}} A_{ \pm} \\
k_{\theta \theta} & =\frac{a \dot{a}}{(-\Delta)^{1 / 2}} \sin ^{2} \chi & K_{\theta \theta}=\frac{a \dot{t}}{(-\Delta)^{1 / 2}} A_{ \pm} \sin ^{2} \chi \\
k_{\phi \phi} & =\frac{a \dot{a}}{(-\Delta)^{1 / 2}} \sin ^{2} \chi \sin ^{2} \theta & K_{\phi \phi} & =\frac{a \dot{t}}{(-\Delta)^{1 / 2}} A_{ \pm} \sin ^{2} \chi \sin ^{2} \theta
\end{array}
$$

It is a straightforward task compute the tensor (3.9) for the present case, which give us

$$
(\Theta)_{\nu}^{\mu}=\left(\begin{array}{ccc}
0 & 0 & 0 \\
0 & \frac{6}{a^{2}} A_{ \pm} & 0 \\
0 & 0 & 0_{3 \times 3}
\end{array}\right)_{5 \times 5} .
$$

The next task is compute the matrix $\Psi$ so, in order to know $\Psi$ is necessary evaluate $\lambda$. It is easily calculated from the relation (3.13), given by

$$
\lambda=-\frac{1}{2 a^{2}}\left(6+\Lambda_{b} a^{2}+\frac{6 \dot{a}^{2}}{(-\Delta)}\right)
$$

This seems contradict the functional dependence for the field previously assumed, but we are free to implement an artistry to convert the velocity dependence to the right 
form by means of the generalized evolution equation, $\left(\dot{a}^{2}+1\right) / a^{2}=\Upsilon H^{2}$, avoiding any misunderstanding.

We turn now to compute a first integral for our specific model. This is performed from (3.11) by setting up $P_{0}$ proportional to the brane energy, $P_{0}:=3 E \Phi=3 E\left(\sin ^{2} \chi \sin \theta\right)$. Furthermore, since we have a homogeneous isotropic space in (4.2), we can invoke the typical value $A_{0 \chi \theta \phi}=\frac{F}{4} a^{4} \Phi$ for the gauge field, which is supported by some kind of cosmological solutions [23, 38, where $\mathrm{F}$ is a constant and the corresponding gauge independent field tensor $F_{\mu \nu \rho \delta \gamma}=5 \nabla_{[\mu} A_{\nu \rho \delta \gamma]}$ is expressed in terms of it $F_{\mu \nu \rho \delta \gamma}=F \epsilon_{\mu \nu \rho \delta \gamma}$. Explicitly, we have

$$
P_{0}=\frac{3 k_{1} a \dot{t} \Phi A_{ \pm}}{\sqrt{-\Delta}}\left(1+\frac{\Lambda_{b}}{6} a^{2}+\frac{\dot{a}^{2}}{(-\Delta)}\right)+\frac{k_{2} F}{4} a^{4} \Phi .
$$

Now, taking into account the generalized evolution equation and $\Lambda_{b}$ being the cosmological constant on the brane, we find the desired result

$$
E=M_{(4)}^{2} a^{4} H^{3}\left(\Upsilon-\frac{\Lambda}{6 H^{2}}\right)^{1 / 2}(\Upsilon-1)+\frac{k_{2} F}{12} a^{4},
$$

where $\Lambda$ is the cosmological constant living in the bulk appearing in Eq. (2.7) and we have used the cosmic gauge in the last step. Note that (4.6) is in keep with Eq. (2.15), confirming equivalence with the limit process developed in Sect. 2.

\section{$5 \quad$ Wheeler-DeWitt equation}

We turn now in this section to develop the quantum description for our specific problem. The canonical quantization procedure is well known so, just remain apply the recipe in the matter of our case.

We shall set $P_{\mu} \rightarrow-i \frac{\delta}{\delta X^{\mu}}$ in such a way that scalar constraints (3.15) and (3.16) transform into quantum equations

$$
\begin{aligned}
& \left(-i \frac{\delta}{\delta X^{\mu}}-p_{A \mu}\right)\left(\Psi^{-1}\right)^{\mu \nu}\left(-i \frac{\delta}{\delta X^{\mu}}-p_{A \mu}\right) \psi=-h \lambda_{0} k_{1}^{2} \psi, \\
& \left(-i \frac{\delta}{\delta X^{\mu}}-p_{A \mu}\right)\left(\Psi^{-2}\right)^{\mu \nu}\left(-i \frac{\delta}{\delta X^{\nu}}-p_{A \nu}\right) \psi=-h k_{1}^{2} \psi,
\end{aligned}
$$

where we have defined $p_{A \mu}:=k_{2} A_{\mu \alpha \beta \gamma} \bar{\varepsilon}^{\alpha \beta \gamma} / 3$ ! .

Specializing to the embedding (4.1) and having in mind the matrix (B.2) in the cosmic gauge, we are able to get the inverse matrix

$$
\left(\Psi^{-1}\right)^{\mu}{ }_{\nu} \equiv\left(\begin{array}{ccc}
A & 0 & 0 \\
0 & B & 0 \\
0 & 0 & N_{3 \times 3}^{-1}
\end{array}\right)=\left(\begin{array}{ccc}
\frac{-1}{3 H^{2}(1-\Upsilon)} & 0 & 0 \\
0 & \frac{a^{2}}{3\left[-H^{2} a^{2}(1-\Upsilon)+2 A_{ \pm}\right]} & 0 \\
0 & 0 & N_{3 \times 3}^{-1}
\end{array}\right)
$$


in such a way that (5.1) and (5.2) transform in the pair of relations

$$
\begin{aligned}
-A_{ \pm}^{-1} A \widetilde{P}_{0}^{2} \psi+A_{ \pm} B \widetilde{P}_{1}^{2} \psi & =-h \lambda_{0} k_{1}^{2} \psi \\
-A_{ \pm}^{-1} A^{2} \widetilde{P}_{0}^{2} \psi+A_{ \pm} B^{2} \widetilde{P}_{1}^{2} \psi & =-h k_{1}^{2} \psi
\end{aligned}
$$

where we introduce the notation $\widetilde{P}_{\mu}=-i \frac{\delta}{\delta X^{\mu}}-p_{A \mu}$. Taking into account the value $\lambda_{0}=$ $3\left[-H^{2}(1+\Upsilon)+\frac{2}{a^{2}}\right]$ expressed in the cosmic gauge, the couple of quantum relations can be rewritten as,

$$
\begin{aligned}
& \widetilde{P}_{0}^{2} \psi=k_{1}^{2}(3 \Phi)^{2} a^{8} H^{6}(1-\Upsilon)^{2}\left(\Upsilon-\frac{\Lambda}{6 H^{2}}\right) \psi, \\
& \widetilde{P}_{1}^{2} \psi=-k_{1}^{2}(3 \Phi)^{2} a^{2} \frac{\left(1-H^{2} \Upsilon a^{2}\right)\left[H^{2} a^{2}(1-\Upsilon)-2+\frac{\Lambda a^{2}}{3}\right]^{2}}{\left(1-\frac{\Lambda a^{2}}{6}\right)^{2}} \psi .
\end{aligned}
$$

At this time, we are more interested in identify the potential governing the dynamics of our model instead of solve exactly the WdW equation so, to get insight we propose the wave function of separable form, $\psi(t, a)=\psi_{1}(t) \Psi(a)$. The WdW equation adquires the form

$$
-\frac{\partial^{2} \Psi}{\partial a^{2}}=\frac{a^{2} M_{(4)}^{4}\left[2-\frac{\Lambda a^{2}}{3}+(\Upsilon-1) H^{2} a^{2}\right]^{2}\left(-1+\Upsilon H^{2} a^{2}\right)}{\left(1-\frac{\Lambda a^{2}}{6}\right)^{2}} \Psi,
$$

accompanied by the energy equation

$$
\left(E-\frac{k_{2} F}{12} a^{4}\right)^{2}=H^{6} a^{8} M_{(4)}^{4}(1-\Upsilon)^{2}\left(\Upsilon-\frac{\Lambda}{6 H^{2}}\right)
$$

where, as before, we have assumed $\widetilde{P}_{0}=(3 \Phi) E$.

\section{Nucleation Rate}

At this stage, we are ready to compute the creation probability which the universe could be created. Some simplifications are necessary due to the general problem itself is hard to solve.

From WdW equation (5.8), is easily read off the potential which is subjected the model (3.1)

$$
V(a)=\frac{a^{2} M_{(4)}^{4}\left[2-\frac{\Lambda a^{2}}{3}+(\Upsilon-1) H^{2} a^{2}\right]^{2}\left(1-\Upsilon H^{2} a^{2}\right)}{\left(1-\frac{\Lambda a^{2}}{6}\right)^{2}} .
$$

Note that this is a very hard expression to work out if one is interested in the general integration, specially if, in the cosmological context, creation probability is desire computed. Recall that the last is written in terms of the potential extracted from the WdW 
equation, namely,

$$
\mathcal{P} \sim e^{-2 \int_{a_{l}}^{a_{r}} \sqrt{V} d a}
$$

In order to get some interesting results from the quantum approach, we shall consider some special cases.

\subsection{Case A}

If $E=0$ from Eq. (5.9) then $\Upsilon$ is just a constant given by

$$
\frac{\left(k_{2} F / 12 M_{(2)}^{2}\right)^{2}}{H^{6}}=(1-\Upsilon)^{2}\left(\Upsilon-\frac{\Lambda}{6 H^{2}}\right) .
$$

The probability rate in this case is

$$
\mathcal{P} \sim e^{\frac{4\left((\Upsilon-1)-\Lambda / 3 H^{2}\right)}{\Upsilon \Lambda}+2(\Upsilon-1) H^{2}\left(\frac{6}{\Lambda}\right)^{2}\left[1-\frac{1}{X} \tan ^{-1} X\right]},
$$

where $X^{2}=\left(\frac{\Lambda}{6 H^{2}}\right)^{2}\left(\Upsilon-\frac{\Lambda}{6 H^{2}}\right)^{-1}$. Now, if $k_{2} F, \Lambda<<H^{2}$ and, at first order the probality rate is

$$
\mathcal{P} \sim e^{-\frac{4}{3 H^{2}}+\frac{16 k_{2} F}{15 H^{5}}}
$$

This means that it is more probable to create a universe when $k_{2} F>0$ than $k_{2} F<0$. We will comment about it below.

Now, we would like calculate the probability nucleation using the instanton method.

The corresponding Euclidean action in de Sitter bulk can be found by complexifying the temporal coordinate and keeping the field strength $F_{\mu \nu \rho \delta \gamma}$ fixed

$$
S_{(E)}=\int_{m} d^{4} x \sqrt{-\gamma}\left(-\frac{M_{(2)}^{2}}{2} \mathcal{R}+\rho_{v}\right)+\frac{k_{2}}{4 !} \int_{m} d^{4} x \sqrt{-\gamma} A_{\mu \nu \rho \sigma} \epsilon^{\mu \nu \rho \sigma} .
$$

In Euclidean space we have now closed worldsheets that split the deSitter background spacetime of radius $H_{d S}^{-1}=(\Lambda / 6)^{-1 / 2}$ in two regions. This is the basic geometry of the instanton calculation.

Following [27, by using Stoke's theorem we can transform (6.6) to an instanton action that involves a volume of the spacetime enclosed by the brane

$$
S_{(E)}=\int_{m} d^{4} x \sqrt{-\gamma}\left(-\frac{M_{(2)}^{2}}{2} \mathcal{R}+\rho_{v}\right)-k_{2} F \int_{v} d^{5} x \sqrt{-g} .
$$

For spherical worlsheets the former action is expressed through the radius $R_{0}$ of the brane

$$
S_{(E)}=\left(\rho_{v}-\frac{12 M_{(4)}^{2}}{R^{2}}\right) S_{4}\left(R_{0}\right)-k_{2} F V_{4}\left(R_{0}\right)
$$


where

$$
S_{(4)}=\frac{8 \pi^{2}}{3} R_{0}^{4}
$$

is the surface of a worldsheet of radius $R_{0}$, and

$$
V_{4}=\pi^{2} H_{d S}^{-5} \phi_{0}-\frac{\pi^{2} H_{d S}^{-4}}{R_{0}}\left(1-R_{0} H_{d S}\right)^{1 / 2}\left(1+\frac{2}{3} R_{0}\right),
$$

is the volume enclosed by the brane of radius $R_{0}$ and $\sin \left(\phi_{0}\right)=R_{0} H_{d S}$. Extremizing (6.8) we find that the radius of the Euclidean brane is a solution of

$$
M_{(4)}^{2} H^{3}\left(\Upsilon-\frac{\Lambda}{6 H^{2}}\right)^{1 / 2}(1-\Upsilon)=\frac{k_{2} F}{12}
$$

where $\Upsilon \equiv H_{d S}^{2}\left(R_{0} H\right)^{-2}$. The resulting Euclidean action is

$$
S_{(E)}=-6 \pi^{2} M_{(4)}^{2}\left\{\frac{4\left[(\Upsilon-1)-\frac{\Lambda}{3 H^{2}}\right]}{\Upsilon \Lambda}+2(\Upsilon-1)\left(\frac{6 H}{\Lambda}\right)^{2}\left[1-\frac{1}{X} \tan ^{-1} X\right]\right\},
$$

and the nucleation probability $\mathcal{P} \sim e^{-S_{(E)}}$ is in agreement with (6.4) modulo a normalizing factor. We now go back to the meaning of equation (6.5). The behavior of strength field $F_{\mu \nu \rho \delta \gamma}$ is the key, when $k_{2}>0$ the field decrease in the inside region with respect to its original value and corresponds to screening membrane discuss in [27. When $k_{2}<0$ correspond to antiscreening membrane and the field increase its value, and as it is expected, is less probable to produce such a Universe. This situation is resembled in phenomena of vacuum decay, where ordinary transition from false to true vacuum corresponds to $k_{2}>0$, and the decay of true vacuum, by means of false vaccum bubbles, corresponds to $k_{2}<0$ and $k_{2} F$ represents the difference in energy density between the false and true vacuum.

\subsection{Case B}

We proceed to calculate an approximate expression for the nucleation rate at first order, when both $E$ and $F$ are small. The potential is

$$
V(a)=4 a^{2}\left(1-H^{2} a^{2}-E H-k_{2} F H a^{4}\right)
$$

and the nucleation probability is

$$
\mathcal{P} \sim e^{-\frac{4}{3 H^{2}}+E H^{-1}+\frac{16 k_{2} F}{15 H^{5}}}
$$

in complete agreement with (6.5) when $E$ vanishies.

The potential for case A, is plotted in figure (11) and the corresponding one for the case $\mathrm{B}$ is in figure (21). Using this kind of plots for the potential, we can deduce that creation probability is enhance when the nucleation process take place in de Sitter background spacetime with small radius $H_{d S}^{-1}$. 


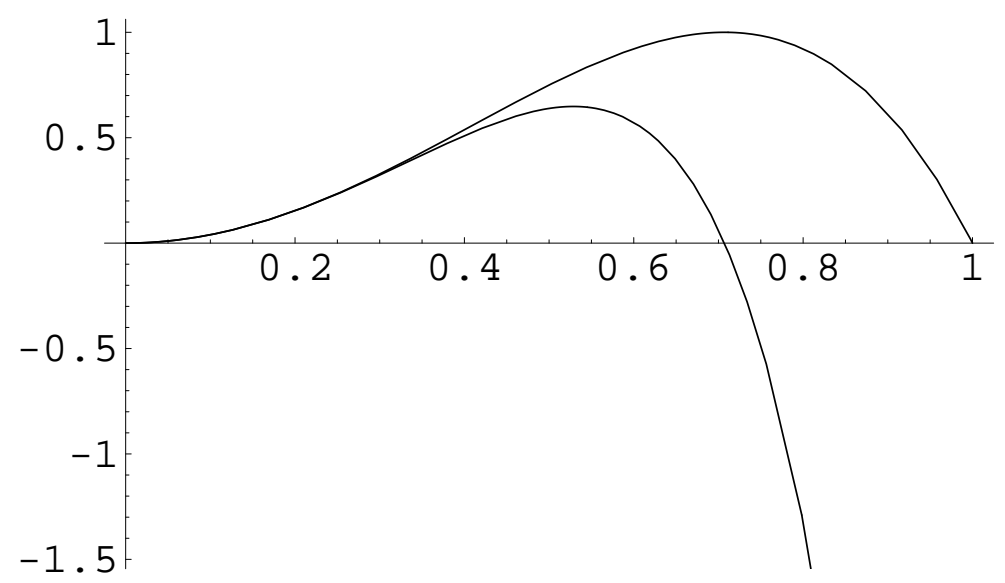

Figure 1: Potential for case A. In this case $E=0$ and $k=k_{2} F$ taking the values: $k=0$ (Einstein case) for the upper curve and $k \neq 0$ for the lower curve.

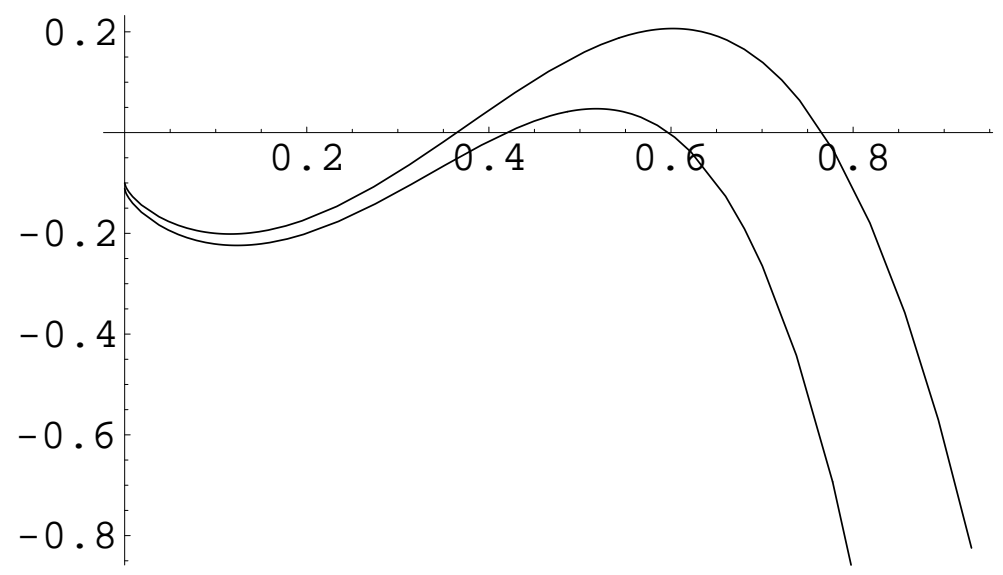

Figure 2: Potential for case B. In this case $E \neq 0$ and the background is a de Sitter space. $k=0$ for the upper curve and $k \neq 0$ for the lower curve.

\section{Conclusions}

We have calculated the nucleation probability of brane world universes induced by a totally antisymmetric tensor living in a dS fixed background spacetime. This was done by means of canonical quantum approach where the Wheeler-DeWitt equation was found. Besides, we found for one specific case, the nucleation rate computing the corresponding instanton. When the energy of the brane $\mathcal{E}=0$ in the bulk space and the coupling constant of the brane $k_{2}$ with the antisymmetric field is positive, the creation probability is enhanced with respect to no interaction of the brane with the 4 -form. For $k_{2}<0$ the nucleation rate decresed as is expected. This situation is resembled in phenomena of vacuum decay, where ordinary transition from false to true vacuum corresponds to 
$k_{2}>0$, and the decay of true vacuum by means of false vaccum bubbles corresponds to $k_{2}<0$. Furthermore, $k_{2} F$ represents the difference in energy density between the false and true vacuum.

For large expansion rate of the de Sitter bulk we observed an increase nucleation rate. At this point we ask ourselves about possible brane collisions, and what the most important factor in this issue is. The branes will be driven apart by the exponential expansion of the bulk reducing brane collision but at the same time, there is an increase in nucleation rate. We expect now that the problem of old inflationary model of the universe is an advantage: bubbles may not be produced fast enough, to complete cover the bulk.

Once the brane universe was created it still could be hitting by stealth branes [32], that by means of constraining some parameters of the model reduce the rate of brane collisions to an acceptable level. We think that cosmological constraints can impose bounds on the values of $k_{2} F$ and with this value one could try to answer the question: Is our universe very special?

\section{Acknowledgements}

We benefited from Germán Mandujano for assistance. ER would like to thank César de la Cruz, Raúl Hernández, Carlos Vargas and Alfredo Villegas for useful discussions and encourage the paper. We also thank to SNI-México for partial support.

\section{Appendix A}

\section{Embedding theory}

Consider a brane, $\Sigma$, of dimension $d$ whose worldsheet, $m$ is an oriented timelike manifold living in a $N$-dimensional arbitrary fixed background spacetime $M$ with metric $g_{\mu \nu}$. For hamiltonian purposes, we shall foliate the worldsheet $m$ in spacelike leaves $\Sigma_{t}$.

Taking advantage of the differential geometry for surfaces, as well as novelty variational techniques developed in [39, 40] we can write the Gauss-Weingarten equations associated with the embedding of $\Sigma_{t}$ in $M\left(x^{\mu}=X^{\mu}\left(u^{A}\right)\right)$, i.e., the gradients of the $\Sigma_{t}$ basis $\left\{\epsilon_{A}^{\mu}, \eta^{\mu}, n^{\mu}{ }_{i}\right\}$. These spacetime vectors can be decomposed with respect to the adapted basis to $\Sigma_{t}$, as

$$
\begin{aligned}
\mathcal{D}_{A} \epsilon^{\mu}{ }_{A} & =-\Gamma_{\alpha \beta}^{\mu} \epsilon_{A}^{\alpha} \epsilon_{B}^{\beta}+k_{A B} \eta^{\mu}-K_{A B}^{i} n^{\mu}{ }_{i} \\
\mathcal{D}_{A} \eta^{\mu} & =k_{A B} \epsilon^{\mu B}-K_{A}{ }^{i} n^{\mu}{ }_{i} \\
\tilde{\mathcal{D}}_{A} n^{\mu i} & =K_{A B}^{i} \epsilon^{\mu B}-K_{A}{ }^{i} \eta^{\mu}
\end{aligned}
$$

where $\Gamma_{\beta \gamma}^{\alpha}$ are the Christoffel coefficients of the background manifold and, $K_{A}{ }^{i}$ is a piece of the generalized extrinsic twist potential and both $k_{A B}$ and $K_{A B}^{i}$ are the extrinsic 
curvatures of $\Sigma_{t}$ associated with the normals $\eta^{\mu}$ and $n^{\mu}{ }_{i}$, respectively. $\mathcal{D}_{A}$ denotes the covariant derivative adapted to $\Sigma_{t}$ and $\tilde{\mathcal{D}}_{a}$ is the covariant derivative that preserves invariance under rotations of the normals $n^{\mu}{ }_{i}$, i.e., $\tilde{\mathcal{D}}_{A}^{i}=\mathcal{D}_{A}^{i}-\omega_{A}^{i j} n_{j}$. In a similar way, we can write the Gauss-Weingarten equations associated with the embedding of $\Sigma_{t}$ in the worldsheet $m,\left(x^{a}=X^{a}\left(u^{A}\right)\right)$, i.e., the gradients of the $\Sigma_{t}$ basis $\left\{\epsilon^{a}{ }_{A}, \eta^{a}\right\}$. These worldsheet vectors can be decomposed with respect to the adapted basis to $\Sigma_{t}$, as

$$
\begin{aligned}
\nabla_{A} \epsilon_{B}^{a} & =\gamma_{A B}^{C} \epsilon_{C}^{a}+k_{A B} \eta^{a} \\
\nabla_{A} \eta^{a} & =k_{A B} \epsilon^{a B}
\end{aligned}
$$

where $\nabla_{A}$ is the gradient along the tangent basis, i.e., $\nabla_{A}=\epsilon^{a}{ }_{A} \nabla_{a}$, where $\nabla_{a}$ is the covariant derivative compatible with $\gamma_{a b}$.

The time vector field, written in terms of the adapted basis of a leaf $\Sigma_{t}$, is given by

$$
t^{\mu}=\dot{X}^{\mu}=N \eta^{\mu}+N^{A} \epsilon_{A}^{\mu}
$$

which represents the flow of time throughout spacetime. Note that we are able to rewrite the previous time deformation vector as follows

$$
\begin{aligned}
\nabla X^{\mu} & :=t^{a} \nabla_{a} X^{\mu}-N^{A} \mathcal{D}_{A} X^{\mu} \\
& =N \eta^{\mu},
\end{aligned}
$$

where, taking into account the well known notation, $\nabla_{a}$ denotes the covariant derivative compatible with $\gamma_{a b} \quad(\mu, \nu=0,1,2, \ldots, N-1 ; a, b=0,1, \ldots, d$ and $A, B=1,2, \ldots, d)$. Furthermore, from (A.6) note that the following relations hold:

$$
N=-g_{\mu \nu} \eta^{\mu} \dot{X}^{\mu} \quad \text { and } \quad N^{A}=g_{\mu \nu} h^{A B} \epsilon_{A}^{\mu} \dot{X}^{\nu}
$$

\section{Appendix B}

\section{$\Psi \quad$ Matrix}

In this appendix we write the full matrix $\Psi$ for our embedding (4.1). Taking into account the Eq. (4.3) as well as Eq. (4.4) we have

$$
(\Psi)^{\mu \nu}=\left(\begin{array}{cccc}
-\frac{1}{2 a^{2} A_{ \pm}}\left[6+\Lambda_{b} a^{2}+\frac{6 \dot{a}^{2}}{(-\Delta)}\right] & 0 & 0 & 0 \\
0 & \frac{A_{ \pm}}{2 a^{2}}\left[6+\Lambda_{b} a^{2}+\frac{6 \dot{a}^{2}}{(-\Delta)}+12 A_{ \pm}\right] & 0 & 0 \\
0 & 0 & \frac{1}{2 a^{4}\left[6+\Lambda_{b} a^{2}+\frac{6 \dot{a}^{2}}{(-\Delta)}\right]} & 0 \\
0 & 0 & 0 & M_{2 \times 2}
\end{array}\right) .
$$

The previous matrix, in the cosmic gauge, reduces to a more manageable form

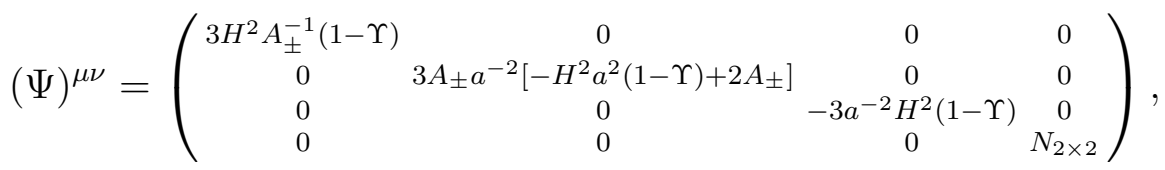

where $M_{2 \times 2}$ and $N_{2 \times 2}$ denote $2 \times 2$ diagonal matrices. 


\section{REFERENCES}

[1] A. Vilenkin, Phys.Lett B 117, 25 (1982), Phys. Rev. D 30, 509 (1984); Phys. Rev. D 33, 3560 (1986).

[2] J. B. Hartle and S. W. Hawking, Phys. Rev. D 28, 2960 (1983).

[3] A. D. Linde, Lett. Nuovo Cimento 39, 401 (1984).

[4] V. A. Rubakov, Phys. Lett. B 148, 280 (1984).

[5] K. V. Kuchař, The Problem of Time in Canonical Quantization, in Conceptual Problems of Quantum Gravity, Eds. A. Ashtekar and J. Stachel, Birkhäuser, Boston, (1991);

[6] J. B. Hartle, Quantum Cosmology: Problems for the $21^{\text {st }}$ Century, gr-qc/9701022.

[7] N. Arkani-Hamed, S. Dimopoulos and G. Dvali, Phys. Lett. B 429, 263 (1998); I. Antoniadis, N. Arkani-Hamed, S. Dimopoulos and G. Dvali, Phys. Lett. B436, 257 (1998).

[8] L. Randall and R. Sundrum, Phys. Rev. Lett 83, 3370 (1999); 4690 (1999).

[9] T. Regge and C. Teitelboim, in Proc. Marcel Grossman, p. 77 (Trieste, 1975);

[10] M. D. Maia and W. Mecklemburg, J. Math. Phys. 253047 (1984); M. D. Maia, Phys. Rev. D 31262 (1985);

[11] M. Pavsic, Phys. Lett. A 1161 (1986);

[12] M. Pavsic and V. Tapia, Resource Letter on Geometrical Results for Embeddings and Branes, gr-qc/0010045;

[13] V. A. Rubakov, Large and Infinite Extra Dimensions, hep-th/0104152;

[14] Supernova Cosmology Project, http://www.supernova.lbl.gov

[15] G. Dvali, G. Gabadadze and M. Porrati, Phys. Lett. B 484, 112 (2000).

[16] C. Deffayet, Phys. Lett. B 502, 199 (2001).

[17] G. Dvali, G. Gabadadze, Phys. Rev. D 63, 065007 (2001).

[18] P. Binetruy, C. Defayyet, and D. Langlois Nucl. Phys. B 565, 269 (2000).

[19] D. Ida, JHEP 0009, 014 (2000). 
[20] N. Deruelle and T. Dolezel, Phys. Rev. D 62, 103502 (2001).

[21] P. Bowcock, C. Charmousis and R. Gregory, Class. Quant. Grav. 17, 4745 (2000).

[22] A. C. Davis, S. C. Davis, W. B. Perkins and I. R. Vernon, Phys.Lett B 504, 254 (2001).

[23] B. Carter and J.P. Uzan, gr-qc/0101010; R. Battye, B. Carter, A. Mennim and J.P. Uzan, hep-th/0105091; B. Carter, J. P. Uzan, R. A. Battye and A. Mennim, Class. Q. Grav. 184871 (2001);

[24] L. Anchordoqui, C. Nuñez and K. Olsen, hep-th/0007064.

[25] H. Collins and B. Holdom, Phys. Rev. D 62, 105009 (2000); 62, 124008 (2000).

[26] J. D. Brown and C. Teitelboim, Nucl. Phys. B 297787 (1988);

[27] J. Garriga Phys. Rev. D 49, 6327 (1994).

[28] J. Garriga and M. Sasaki, Phys. Rev. D 62, 043523 (2000).

[29] A. Gorsky and K. Selivanov, Phys. Lett. B 485, 271 (2000);

[30] S. Nojiri and S.D. Odintsov, JHEP 12, 033 (2001);

[31] M. Bouhmadi-López, P. F. González-Díaz and A. Zhuk, Class. Quant. Grav. 19, 4863 (2002);

[32] R. Cordero and A. Vilenkin, Phys. Rev. D 65083519 (2002);

[33] D. Karasik and A. Davidson, Geodetic Brane Gravity, gr-qc/0207061; Mod. Phys. Lett. A 132187 (1998);

[34] R. Cordero, and E. Rojas, Phys. Lett. 47045 (1999);

[35] R. Capovilla, J. Guven and E. Rojas Nucl. Phys. Proc. Suppl. 88337 (2000);

[36] R. Cordero, and E. Rojas Int. Journal of Mod. Phys. A 1773 (2002);

[37] R. M. Wald, General Relativity, University of Chicago (1986);

[38] A. Kehagias and E. Kiritsis, JHEP 9911022 (1999);

[39] R. Capovilla and J. Guven, Phys. Rev. D 516736 (1995); Phys. Rev. D 575158 (1998);

[40] J. Guven, Phys. Rev. D 485562 (1993); Phys. Rev. D 484604 (1993); 